\title{
Relativistically correct hole-boring and ion acceleration by circularly polarized laser pulses
}

\author{
A P L Robinson ${ }^{1}$, P Gibbon ${ }^{2}$, M Zepf $^{3}$, S Kar ${ }^{3}$, R G Evans $^{4}$ and C Bellei $^{4}$ \\ ${ }^{1}$ Central Laser Facility, STFC Rutherford-Appleton Laboratory, Chilton, Didcot, Oxfordshire, \\ OX11 0QX, UK \\ 2 Jülich Supercomputing Centre, Institute for Advanced Simulation, Forschungzentrum Jülich \\ GmbH, D-52425, Jülich, Germany \\ ${ }^{3}$ Department of Physics and Astronomy, Queen's University Belfast, BT7 1NN, UK \\ ${ }^{4}$ Plasma Physics Group, Blackett Laboratory, Imperial College London, SW7 2BZ, UK
}

Received 28 April 2008, in final form 1 August 2008

Published 7 January 2009

Online at stacks.iop.org/PPCF/51/024004

\begin{abstract}
The problem of the 'hole-boring' (HB)-type of radiation pressure acceleration of ions by circularly polarized laser pulses interacting with overdense plasmas is considered in the regime where the dimensionless scaling parameter $I / \rho c^{3}$ becomes large. In this regime a non-relativistic treatment of the 'HB' problem is no longer adequate. A new set of fully relativistic formulae for the mean ion energy and ' $\mathrm{HB}$ ' velocity is derived and validated against one-dimensional particle-in-cell simulations. It is also found that the finite acceleration time of the ions results in large energy spreads in the accelerated ion beam even under the highly idealized conditions of constant laser intensity and uniform mass density.
\end{abstract}

(Some figures in this article are in colour only in the electronic version)

\section{Introduction}

Recently there has been considerable interest in laser-acceleration of protons and ions by the radiation pressure acceleration (RPA) (or radiation pressure dominant (RPD)) mechanism using short-pulse laser systems reaching intensities in excess of $10^{20} \mathrm{~W} \mathrm{~cm}^{-2}$ [1-12]. RPA has attracted this attention because it opens up the possibility of reaching very high ion energies $(>100 \mathrm{MeV})$, very high conversion efficiencies $(>10 \%)$, and the production of quasimonoenergetic beams. There has been particular interest in RPA with circularly polarized pulses as this suppresses fast electron generation and leads to the interaction being dominated by the radiation pressure [3].

It has been shown that there are two distinct modes in which RPA operates. Firstly, there is the mode in which the laser pulse interacts with a semi-infinite target, i.e. driving material ahead of it as a piston but without interacting with the target rear surface. This might be termed the 'hole-boring' (HB) mode of RPA [1-7, 12-15]. 
Secondly, there is an alternative scenario which might be termed the 'light-sail' (LS) mode of RPA. This occurs if the target is sufficiently thin for the laser pulse to punch through the target and accelerate a slab of plasma as a single object $[8-11,16,17]$. The underlying physics has some similarities to that of the 'LS' concept of space-flight $[18,19]$. More than one recent publication has shown that the results of particle-in-cell (PIC) simulations of the LS scenario can be very well modelled in one dimension, by treating the plasma slab as a single object which perfectly reflects the incident light $[8,10]$. This reduces the problem to a single equation of motion,

$$
\frac{\mathrm{d} p}{\mathrm{~d} t}=\frac{2 I}{c} \frac{\sqrt{p^{2}+\sigma^{2} c^{2}}-p}{\sqrt{p^{2}+\sigma^{2} c^{2}}+p},
$$

plus an equation to specify the incident light intensity, $I$. In equation (1) $\sigma=\rho l$ (where $l$ is the foil thickness). Since this model is fully relativistically correct, it correctly describes the acceleration of ions to $\mathrm{GeV}$ energies and agrees with PIC simulations. In contrast, a number of publications that are concerned with the HB mode of RPA make use of non-relativistic expressions (for example $[1,3,5,11]$ ). This is only really acceptable for low values of $I / \rho$ for which the HB velocity, $v_{\mathrm{b}}$, is small (i.e. $v_{\mathrm{b}} \ll c$ ). The development of 'extreme' intensity systems which can achieve intensities in excess of $10^{22} \mathrm{~W} \mathrm{~cm}^{-2}$ over the next decade will mean that it is possible to access regimes where the non-relativistic theory of HB is no longer valid.

There are a number of other issues in the study of both HB and LS RPA that are the subject of current research. This includes transverse instabilities in LS RPA [10,9], the effect of multiple ion species (both HB and LS) and the effect of the laser spot profile [10,17]. The aforementioned issues are related to the quality of acceleration under realistic 3D conditions. A thorough understanding of these issues is important for exploiting the full potential of RPA.

This paper is primarily concerned with the development of a relativistically correct theory of $\mathrm{HB}$ in one dimension (1D). A new set of expressions for the HB velocity, mean ion energy and conversion efficiency are derived. These expressions are then compared with the results of 1D electromagnetic PIC simulations of circularly polarized laser pulses normally incident on plasma slabs, and it is found that the agreement is very good. The energy spectrum of the accelerated protons is also discussed. Previously, Zhang et al [20] have put forward a 1D relativistic model of $\mathrm{HB}$, the results of which differ somewhat from the model that is presented here (this issue is discussed later).

In general, HB results in substantially lower peak energies that are possible in LS mode and, as we shall show, also tends to produce wider energy distributions. However, HB should be much less sensitive to laser prepulse and spatial intensity variations which may make it an attractive choice for high intensity laser ion acceleration.

The paper is organized as follows: in section 2 the non-relativistic theory of HB is reviewed. In section 3 the new, relativistically correct theory of $\mathrm{HB}$ is developed and a set of new expressions are derived. In section 4 both the non-relativistic and the new relativistic theories are compared with 1D PIC simulations. The findings of this paper are then summarized in section 5.

\section{Non-relativistic theory of HB RPA}

Consider a 1D situation where a beam of light of constant intensity $I$ is driving into a plasma of uniform density and one ion species. It is assumed that the plasma is effectively collisionless, that the light beam is perfectly reflected at the plasma surface, and that relativity can be neglected. The steady state of this system can be found by examining the momentum balance in the instantaneous rest frame (IRF). Let the velocity of the plasma surface in the lab frame 
be $v_{\mathrm{b}}$. The plasma that is at rest in the lab frame now approaches the plasma surface at $-v_{\mathrm{b}}$ in the IRF. In order to conserve the particle number there must therefore be a beam of plasma propagating at $+v_{\mathrm{b}}$ away from the plasma surface. The momentum balance in the IRF is therefore given by

$$
\frac{2 I}{c}=2 n_{\mathrm{i}} m_{\mathrm{i}} v_{\mathrm{b}}^{2}
$$

where $n_{\mathrm{i}}$ is the ion density and $m_{\mathrm{i}}$ is the ion mass. At this point we shall define a dimensionless pistoning parameter,

$$
\Xi=\frac{I}{m_{\mathrm{i}} n_{\mathrm{i}} c^{3}}=\frac{I}{\rho c^{3}},
$$

where $\rho$ is the mass density of the plasma. From equation (2), one can immediately determine $v_{\mathrm{b}}$ :

$$
v_{\mathrm{b}}=\sqrt{\frac{I}{n_{\mathrm{i}} m_{\mathrm{i}} c}}=\sqrt{c^{2} \Xi} .
$$

By making a Gallilean transformation back to the lab frame, the velocity of the accelerated ions must be $v_{\mathrm{i}}=2 v_{\mathrm{b}}$, and the energy of the ions is therefore given by

$$
\epsilon=\frac{2 I}{n_{\mathrm{i}} c}=2 m_{\mathrm{i}} c^{2} \Xi .
$$

It is straightforward to re-arrange the expression that Macchi gives in [3],

$$
\frac{v_{\mathrm{i}, \max }}{c}=2 \sqrt{\frac{Z}{A} \frac{m_{\mathrm{e}}}{m_{\mathrm{p}}} \frac{n_{\mathrm{c}}}{n_{\mathrm{e}}}} a_{\mathrm{L}},
$$

into equation (4). Although Macchi uses a somewhat different model and derivation in [3], momentum conservation in the non-relativistic limit is still central, hence, the equivalence of his result and the one given here. Zhang et al and Wilks et al also use essentially the same expressions in [11] and [1], respectively, with the derivation given here.

One may be tempted to state that all that needs to be done to make this fully relativistic is to change the RHS of equation (2) to $2 \gamma_{\mathrm{b}}^{2} n_{\mathrm{i}} m_{\mathrm{i}} v_{\mathrm{b}}^{2}$ (where $\gamma_{\mathrm{b}}=\left(1-v_{\mathrm{b}}^{2} / c^{2}\right)^{-1 / 2}$ ), find $v_{\mathrm{b}}$ and then use the Lorentz transform to obtain the ion energy in the lab frame. However, one then finds that $v_{\mathrm{b}} / c=\sqrt{\Xi /(1+\Xi)}$, and, on applying the Lorentz transform, one obtains $\epsilon=2 \Xi m_{\mathrm{i}} c^{2}$, i.e. exactly the non-relativistic result! The problem is not that one has treated the RHS incorrectly in doing this, but rather that one has failed to treat the LHS of the momentum balance equation correctly.

\section{Relativistic theory of HB RPA}

The crucial step in developing a fully relativistic 1D theory of HB is to note that the light intensity in the IRF is not equal to the light intensity in the lab frame. In fact, it can be shown that

$$
\frac{I^{\mathrm{IRF}}}{I}=\frac{1-\left(v_{\mathrm{b}} / c\right)}{1+\left(v_{\mathrm{b}} / c\right)} .
$$

This result follows from the relativistic Doppler shift and the conservation of photon number. The reader may note that this factor appears in the RHS of equation (1). Once this is recognized, 
one can proceed to write down the correct expression for the momentum balance in the IRF of the plasma surface,

$$
\frac{2 I}{c}\left(\frac{1-v_{\mathrm{b}} / c}{1+v_{\mathrm{b}} / c}\right)=2 \gamma_{\mathrm{b}}^{2} m_{\mathrm{i}} n_{\mathrm{i}} v_{\mathrm{b}}^{2} .
$$

The problem can now be solved in a purely algebraic fashion. One can re-arrange equation (8) in terms of a dimensionless quadratic equation that only includes $\beta_{\mathrm{b}}=v_{\mathrm{b}} / c$ and $\Xi$,

$$
(\Xi-1) \beta_{\mathrm{b}}^{2}-2 \Xi \beta_{\mathrm{b}}+\Xi=0 .
$$

The root of this that corresponds to the physically correct HB velocity is then

$$
\beta_{\mathrm{b}}=\frac{\sqrt{\Xi}}{1+\sqrt{\Xi}} .
$$

The ion energy in the lab frame can now be calculated by using the appropriate Lorentz transform and expressed purely in terms of $\Xi$ and $m_{\mathrm{i}} c^{2}$, yielding

$$
\varepsilon=m_{\mathrm{i}} c^{2}\left[\frac{1+\beta_{\mathrm{b}}^{2}}{1-\beta_{\mathrm{b}}^{2}}-1\right]
$$

and

$$
\varepsilon=m_{\mathrm{i}} c^{2}\left[\frac{2 \Xi}{1+2 \sqrt{\Xi}}\right] .
$$

If the laser pulse intensity is a 'top-hat' function of time, then one can also derive expressions for the $\mathrm{HB}$ duration, $t_{\mathrm{HB}}$, and the real conversion efficiency, $\chi$. The HB duration is determined by noting that the time at which the tail-edge of the pulse hits the plasma surface is when $v_{\mathrm{b}} t=c t-c t_{\mathrm{L}}$, where $t_{\mathrm{L}}$ is the laser pulse duration. From this one gets,

$$
t_{\mathrm{HB}}=\frac{c t_{\mathrm{L}}}{c-v_{\mathrm{b}}} \text {. }
$$

The real conversion efficiency is determined by first writing

$$
\chi=\frac{v_{\mathrm{b}} t_{\mathrm{b}} n_{\mathrm{i}} \epsilon}{I t_{\mathrm{L}}}
$$

and then re-arranging to express $\chi$ as a function of $\Xi$ alone, yielding

$$
\chi=\frac{2 \sqrt{\Xi}}{1+2 \sqrt{\Xi}} .
$$

Equations (10) and (12) are plotted against equations (4) and (5) in the dimensionless form in figure 1. From figure 1 one can also assess those regimes in which relativistic effects are genuinely important in HB RPA. The most extreme regime is at very high intensity, e.g. $10^{23} \mathrm{~W} \mathrm{~cm}^{-2}$, and at very low mass density, e.g. liquid hydrogen $\left(70.8 \mathrm{~kg} \mathrm{~m}^{-3}\right)$. In this case $\Xi=0.52$, and from figure 1(ii) it can be seen that the relativistic expressions give substantially (a factor of 2) lower values than the non-relativistic expression. If one considers a lower intensity of $I=5 \times 10^{21} \mathrm{~W} \mathrm{~cm}^{-2}$, and the same mass density, then $\Xi=0.026$. From figure 1(iv) it can be seen that the difference between the two expressions for ion energy is 

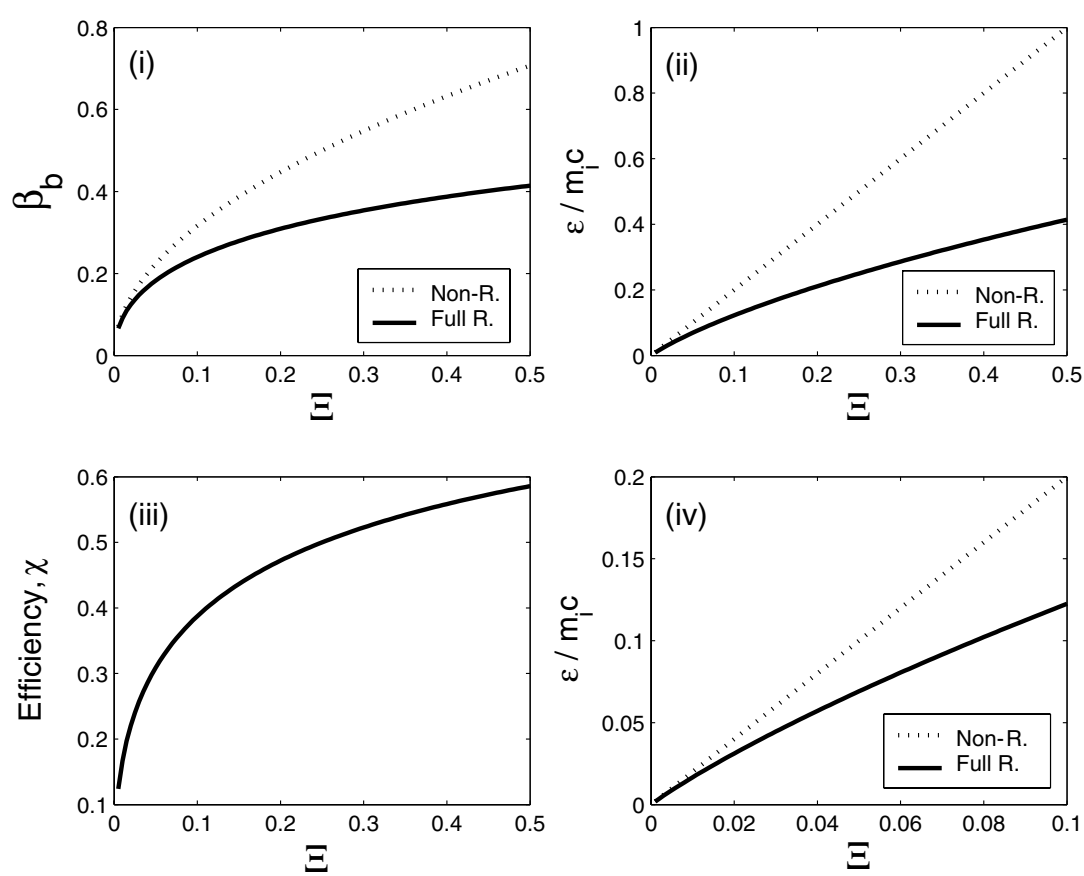

Figure 1. (i) Plot of HB velocity as given by equations (10) (solid) and equation (4) (dotted), (ii) and (iv) plot of accelerated ion energy as given by equation (12) (solid) and equation (5) (dotted) and (iii) plot of conversion efficiency as given by equation (15).

now about $30-40 \%$. On the other hand, a conventional solid target with a mass density of $1000 \mathrm{~kg} \mathrm{~m}^{-3}$ (a C or $\mathrm{CH}$ foil) irradiated at $10^{23} \mathrm{~W} \mathrm{~cm}^{-2}$ corresponds to $\Xi=0.037$. From figure 1(iv) it can again be seen that in this case the difference between the relativistic and nonrelativistic expressions is only $30-40 \%$ even at this extreme intensity. Note that this shows that HB RPA is quite different from LS RPA. In LS RPA the areal mass of the foil, $\sigma$, is important (see equation (1)) not $\rho$. Thus, a dense but thin foil can still be accelerated to velocities at which relativistic effects are most definitely important, as has been shown in another work [10].

One can therefore conclude that the new relativistic formulae are only of significant importance for scenarios in which the dimensionless pistoning parameter $\Xi>0.02$. In practical terms this means that they are important for low mass density targets, such as a liquid hydrogen target $\left(\rho=70.8 \mathrm{~kg} \mathrm{~m}^{-3}\right)$, and laser intensities in excess of $5 \times 10^{21} \mathrm{~W} \mathrm{~cm}^{-2}$. On the other hand, one already knew, from the non-relativistic expressions, that the highest ion energies were to be obtained in this regime. Therefore, one expects this regime to be investigated experimentally.

In [20], Zhang et al put forward a different model from the one which has been described above. Unlike the model presented in this paper, the model of [20] was derived entirely in the lab frame. This model gives a different prediction for the HB velocity (and thus the accelerated ion energy). In the notation used in this paper the expression Zhang et al give for the $\mathrm{HB}$ velocity is

$$
\beta_{\mathrm{b}}=\frac{1}{2}\left(\sqrt{\Xi^{2}+4 \Xi}-\Xi\right) .
$$

Equation (16) gives a somewhat higher value for the HB velocity than equation (10) (the same applies to the ion energy). On comparing the models with numerical simulation (see 
section 4) it was found that there was a very close agreement with the model presented here and that the model of [20] overestimated the HB velocity and mean ion energy (although it was still considerably better than the non-relativistic formulae). A careful examination of the model of [20] reveals that factors of $\left(1-\beta_{\mathrm{b}}\right)$ have been neglected in the energy and momentum fluxes onto the piston head. When these factors are included, the model of [20] produces the same results as the model presented here.

\section{Comparison to numerical simulations}

The new set of formulae need to be tested against numerical simulations. This is because previous numerical studies have shown that the pistoning process is not as ideal as the scenario considered in the analytic model. In particular it has previously been found that the accelerated ions do not constitute a perfect monoenergetic beam $[3,4]$. However if the beam is quasimonoenergetic then the energy of the peak should be close to the analytic prediction. Even if the energy distribution is broad, one would expect the average ion energy to still be fairly close to the analytic prediction.

\subsection{Numerical code}

A set of 1D EM PIC calculations were carried out, the results of which were used as a validation test of the new formulae. In our 'standard' 1D simulation, the target consists of a slab of pure hydrogen plasma at a density of $4 \times 10^{28} \mathrm{~m}^{-3}$ that is either 5 or $20 \mu \mathrm{m}$ thick. The spatial grid consists of 200000 cells each $1 \mathrm{~nm}$ wide. The initial electron temperature is $2 \mathrm{keV}$, the protons are initially cold and both species are represented by 400 macroparticles per cell. The 'laser' pulse is a circularly polarized pulse propagating in the $+x$ direction which is initially situated just to the left of the slab. The laser wavelength is set to $1 \mu \mathrm{m}$. The spatial profile of the fields is given by $\exp \left(-\left(\left(x-x_{0}\right)^{10} / 2\left(c \tau_{\mathrm{p}}\right)^{10}\right)\right)$, where $x_{0}=80 \mu \mathrm{m}$ and $\tau_{\mathrm{p}}=100 \mathrm{fs}$. In terms of intensity this pulse rises to an intensity, $I_{0}$, very rapidly and stays at approximately the same intensity for $33 \mathrm{fs}$ before falling. This pulse provides quasi-constant illumination so that a sensible comparison can made with the analytic model (which assumes constant intensity).

\subsection{Energy scaling for constant intensity}

A series of these simulations were carried out for $I_{0}=1,2,4,6,8,10,20,40$ and $60 \times 10^{21} \mathrm{~W} \mathrm{~cm}^{-2}$. The average energy of the accelerated ions was calculated at $100 \mathrm{fs}$ for each simulation. This set of average ion energies is plotted against the predictions of equations (12) and (5) in figure 2.

From figure 2 it is clear that the average energy of the accelerated ions is in good agreement with the fully relativistic expression given in equation (12) and that the PIC results completely disagree with the non-relativistic expression given in equation (5). The difference between the fully relativistic analytic model and the PIC result in terms of the average energy of the accelerated ions is less than $10 \%$.

The fully relativistic theory also correctly predicts the HB velocity. In figure 3 the position of the $n_{\mathrm{p}}=4 \times 10^{28} \mathrm{~m}^{-3}$ point in four different PIC simulations is plotted against time. Also plotted is a red line which corresponds to a point moving with the HB velocity predicted by equation (10). As can be seen, the agreement is excellent.

In terms of the average ion energy and the HB velocity, it is clear that the fully relativistic theory developed in section 3 provides a correct and accurate description for high $\Xi$ scenarios, whereas the non-relativistic theory does not. 

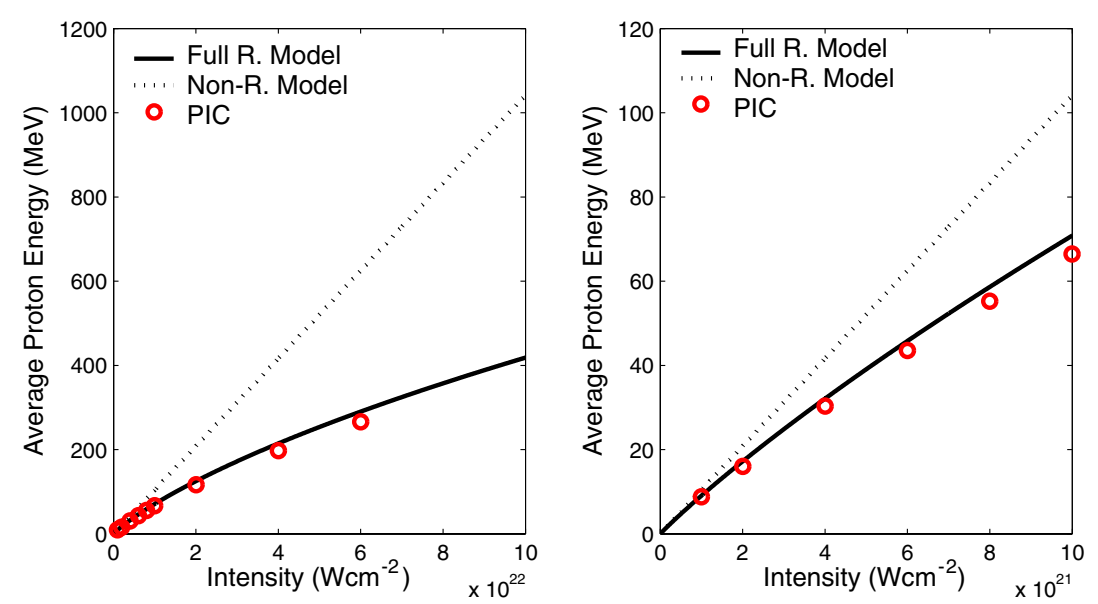

Figure 2. Plots of the mean energy of the accelerated protons in the PIC simulations at $100 \mathrm{fs}$ (circles) and predictions of equation (12) (solid) and equation (5) (dotted).

\subsection{The effect of time-dependent intensity on $H B$}

Since any real laser pulse will generally not be a 'top-hat' function of intensity, the effect of the time dependence needs to be examined. A further PIC simulation which was carried out under the same conditions, but with a temporal profile of the form $I \propto \sin ^{2}(t)$. The full width at half-maximum (FWHM) intensity of the pulse was $1 \times 10^{21} \mathrm{~W} \mathrm{~cm}^{-2}$, and the FWHM pulse duration was $100 \mathrm{fs}$. This simulation can be used as a case study to examine the effects of a time-dependent intensity profile.

The electron population at the front surface will respond to the incident laser pulse on a time-scale comparable to the electron plasma period ( $0.5 \mathrm{fs}$ in this case), whereas the laser intensity varies on a time-scale set by the laser pulse duration ( $100 \mathrm{fs})$. Since the electrons can respond much faster than the laser intensity is varying the electric field at the front surface will change smoothly and the electron momentum balance should be still be maintained to a good approximation. Since the ion acceleration time at the front surface (a few femtoseconds at most) is also significantly lesser than the laser pulse duration, to first approximation the HB process should be a simple convolution of the constant intensity result and the temporal profile of the laser pulse. In the case of the HB velocity this means that equation (10) becomes

$$
\beta_{\mathrm{b}}(t)=\frac{\Xi(I(t))-\sqrt{\Xi(I(t))}}{\Xi(I(t))-1} .
$$

The prediction of equation (17) was compared with the results of the PIC calculation. The analytic prediction was calculated by a simple numerical integration using equation (17), the laser pulse profile used in the PIC simulation and a simple kinematic tracking of the position of the front surface. This was compared with the position of the front surface (in terms of the point where $n_{\mathrm{e}}=40 n_{\mathrm{c}}$ ) from the PIC calculation. The results are plotted in figure 4 .

Figure 4 shows that there is excellent agreement between the 'simple convolution' model and the PIC simulation results. The 'simple convolution' model can also be applied to the mean ion energy. If the HB process is a simple convolution of the constant intensity result and the laser pulse profile, then the total number of ions accelerated per unit area must be given by

$$
N_{\mathrm{acc}}=\int n_{\mathrm{i}} v_{\mathrm{b}}(t) \mathrm{d} t .
$$



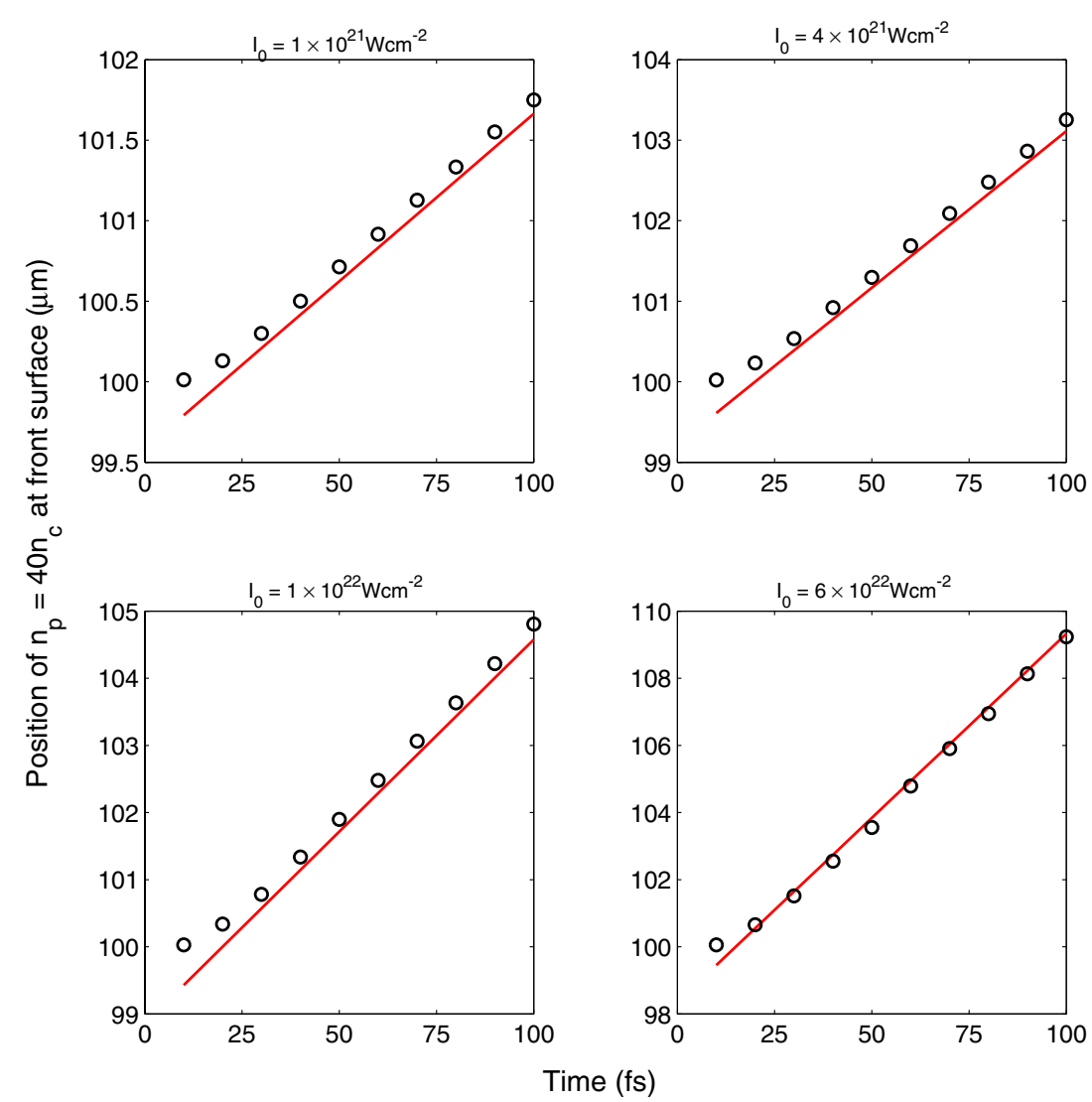

Figure 3. Plots of the position of the $n_{\mathrm{p}}=40 n_{\text {crit }}$ point on the front surface of the target against time in the PIC simulations (intensities indicated above plots). The solid line is the trajectory of a point with a velocity equal to that predicted by equation (10).

The energy that the ions are being accelerated to at any given time will be determined by equation (12), so the mean ion energy then becomes,

$$
\epsilon_{\text {mean }}=\frac{\int n_{\mathrm{i}} v_{\mathrm{b}}(t) \epsilon(t) \mathrm{d} t}{\int n_{\mathrm{i}} v_{\mathrm{b}}(t) \mathrm{d} t .}
$$

If a simple numerical integration is performed to evaluate equation (19) for the same conditions as the PIC simulations then one arrives at a prediction of $11.15 \mathrm{MeV}$. This compares well with the average energy of the accelerated ions in the PIC simulation at $100 \mathrm{fs}$ which is 11.01 MeV. Having examined both average ion energy and the HB velocity, it is therefore not unreasonable to conclude that the 'simple convolution' model represents the HB process well in the case of a time-dependent laser intensity profile.

\subsection{Spectral properties for constant intensity}

The energy spectrum of the accelerated ions is also important, particularly if one has an application of laser acceleration in mind. On the basis of this set of simulations, the shape of the energy spectrum appears to be intensity dependent. The energy spectra of the accelerated protons at $100 \mathrm{fs}$ in the runs where $I_{0}=1,6,10$ and $60 \times 10^{21} \mathrm{~W} \mathrm{~cm}^{-2}$ are shown in figure 5 . 


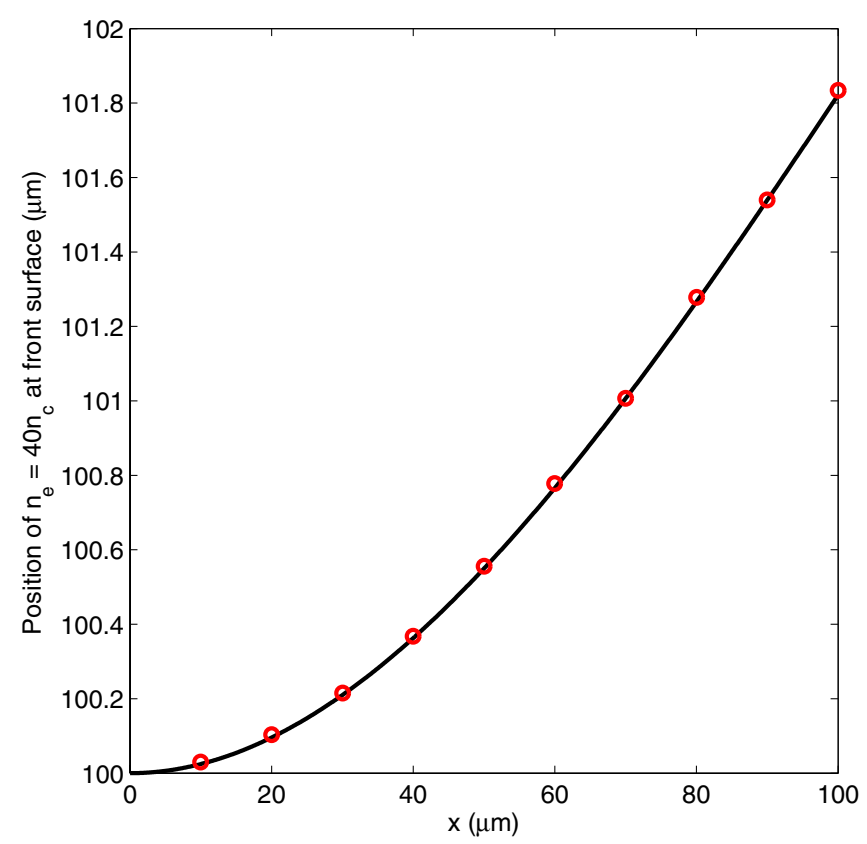

Figure 4. Position of point where $n_{\mathrm{e}}=40 n_{\mathrm{c}}$ at the front surface in the PIC simulations (circles) and an analytic calculation that uses equation (17).

At low intensities the accelerated proton beam is close to being quasi-monoenergetic (as in figure 5(i)). However, at higher intensities (figures 5(ii)-(iv)) the beam has a broad quasiuniform distribution between two cut-offs. Often there are a number of spikes in the spectrum. In $p_{x}-x$ phase space the beam exhibits a lot of fine structures. Examples of this are shown in figure 6.

From figure 6 it can be seen that the fine structure takes the form of a 'ribcage' pattern in $p_{x}-x$ phase space at $100 \mathrm{fs}$. The plots shown in figure 6 at the extremes of the simulation set are representative of the entire set. The physical mechanism that is producing this phase space pattern is the mechanism that is responsible for the energy spread. This behaviour may have been previously observed in the simulations of Zhang et al [20] (see figure 2 of that paper). The cause of this phase space modulation can be seen by looking at a series of plots of the $E_{x}$ component of the electric field. This is shown in figure 7.

Figure 7 shows that the primary spike in the electric field that accelerates the ions at the piston head moves at a steady velocity into the target, as was established by figure 4 . However, the magnitude of the electric field oscillates in time, despite the fact that the laser intensity is approximately constant. It is this oscillation of the magnitude of the electric field that produces the phase space modulation of the accelerated ions. Ahead of the piston head, the fluctuations in the electric field are relatively weak and do not play a significant role in the phase space modulation. In figure 8 the magnitude of this spike in the electric field is plotted against time. This shows that the oscillation has a 'saw-tooth' form.

The large spike in the electric field which is shown in figure 7 is caused by the charge separation at the front surface which in turn is a result of the radiation pressure acting on the electrons. The charge separation region consists of a region consisting almost purely of ions and a region in which there is a surplus of electrons. In figure 9 a plot of the electron and proton densities at the piston head at $50 \mathrm{fs}$ in the simulation with $I_{0}=2 \times 10^{22} \mathrm{~W} \mathrm{~cm}^{-2}$ is 

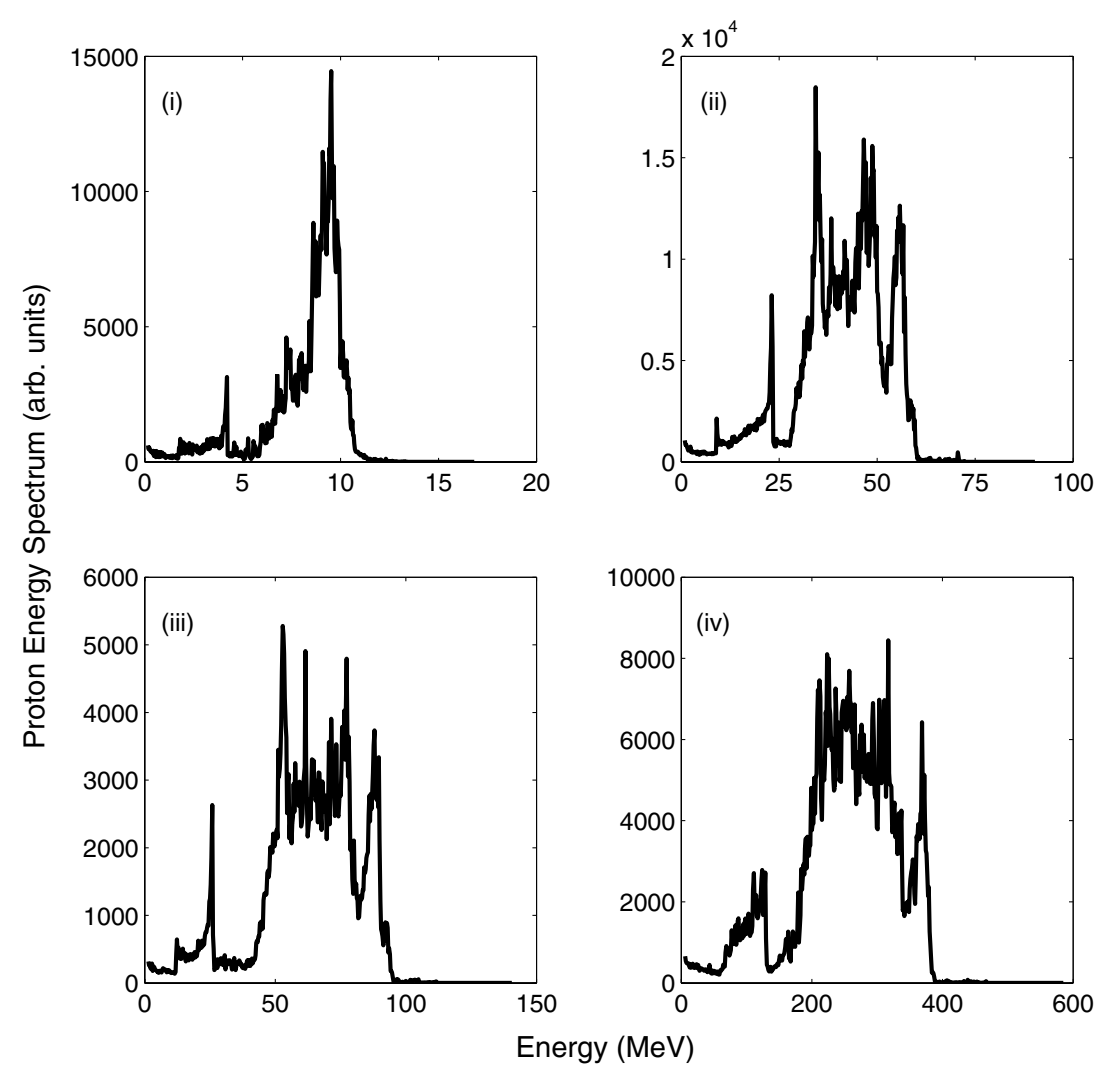

Figure 5. Proton energy spectra of accelerated protons at $100 \mathrm{fs}$ in PIC simulations. (i) $I_{0}=$ $1 \times 10^{21} \mathrm{~W} \mathrm{~cm}^{-2}$, (ii) $I_{0}=6 \times 10^{21} \mathrm{~W} \mathrm{~cm}^{-2}$, (iii) $I_{0}=1 \times 10^{22} \mathrm{~W} \mathrm{~cm}^{-2}$ and (iv) $I_{0}=$ $6 \times 10^{22} \mathrm{~W} \mathrm{~cm}^{-2}$.
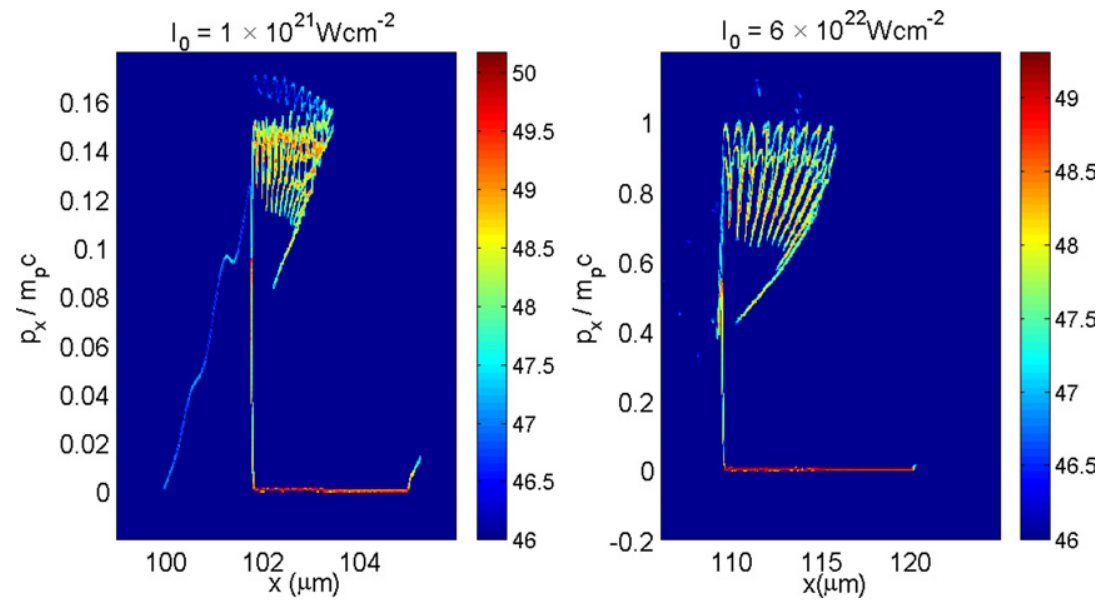

Figure 6. $p_{x}-x$ phase space of protons at $100 \mathrm{fs}$ in PIC simulations (intensities indicated above plots). 


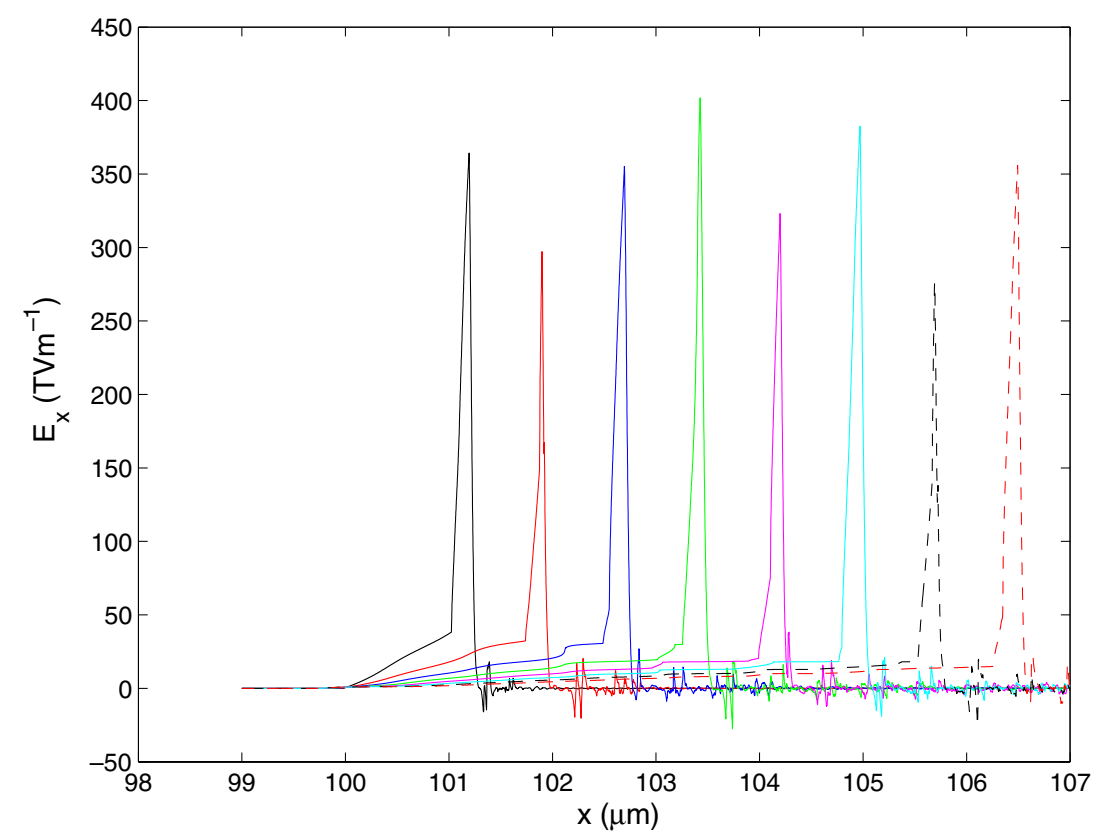

Figure 7. Plots of $E_{x}$ in a simulation with $I_{0}=2 \times 10^{22} \mathrm{~W} \mathrm{~cm}^{-2}$ at $30 \mathrm{fs}$ (black), $40 \mathrm{fs}$ (red), $50 \mathrm{fs}$ (blue), $60 \mathrm{fs}$ (green), $70 \mathrm{fs}$ (magenta), $80 \mathrm{fs}$ (cyan), $90 \mathrm{fs}$ (black dashed) and $100 \mathrm{fs}$ (red dashed).

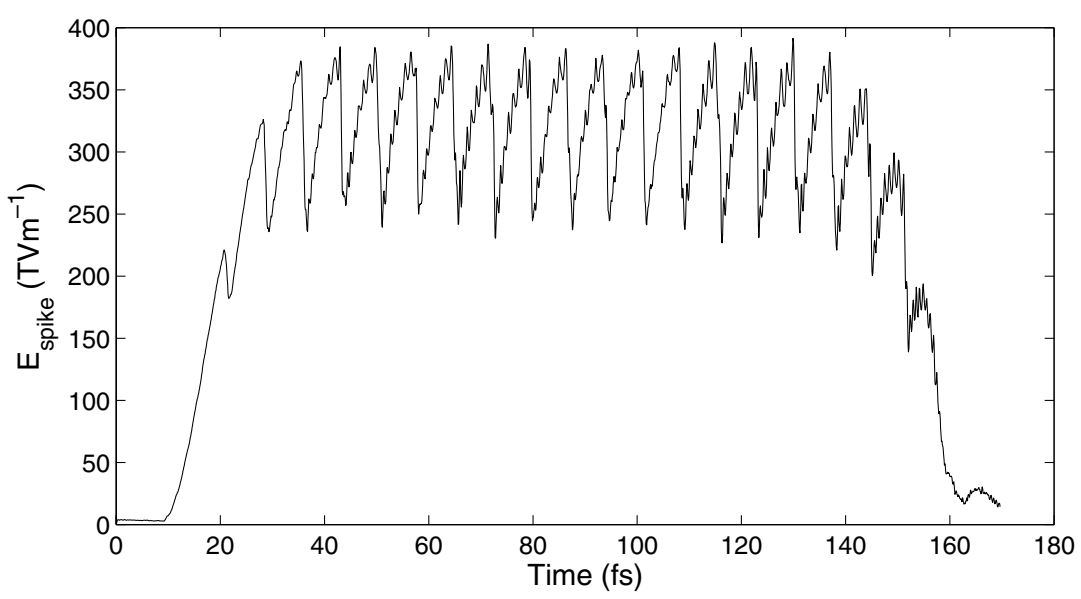

Figure 8. Plot of the magnitude of the main spike in the electric field shown in figure 7 against time.

shown. The positive and negative regions are indicated and the widths of these regions are denoted as $d_{+}$and $d_{-}$, respectively.

Let the proton density in the proton-only region be $n_{+}$, which one can approximate to be a uniform value (we also denote the electron density in the surplus region by $n_{-}$). From Gauss' law one can therefore see that the magnitude of the electric field $\left(E_{x, 0}\right)$ is related to the characteristics of the proton-only region by

$$
E_{x, 0}=\int_{-\infty}^{x_{\text {piston }}} \frac{e n_{\mathrm{p}}}{\epsilon_{0}} \mathrm{~d} x \approx \frac{e n_{+} d_{+}}{\epsilon_{0}} .
$$




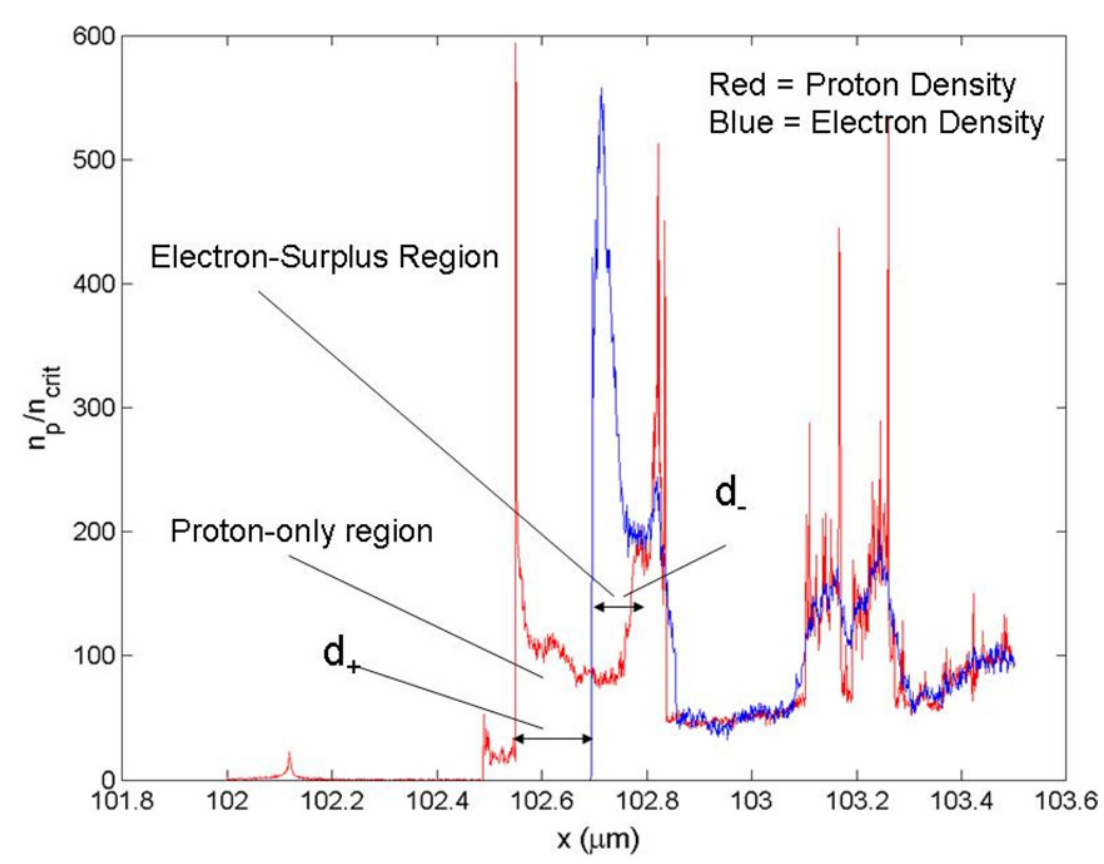

Figure 9. Plots of electron (blue) and proton (red) proton densities at front of the target at $50 \mathrm{fs}$ in simulation with $I_{0}=2 \times 10^{22} \mathrm{~W} \mathrm{~cm}^{-2}$. Features of the charge separation region are indicated.

Equation (20) indicates that if the magnitude of the electric field is oscillating, then this must be because the number of protons per unit area $\left(n_{+} d_{+}\right)$in the proton-only region is also fluctuating, i.e. under- or overcharging is occurring. On examining the PIC simulations it is also clear that this is the case. This indicates that the key to understanding the phase space modulations must be the reason why the number of protons in the charge separation region cannot remain constant to a good approximation.

This is a result of the fact that the laser pulse does not act as an ideal piston. Specifically it does not instantaneously reflect the ions in the rest frame of the piston-head. Instead the protons take a finite time to be accelerated. Therefore, if the piston head recedes and a stationary proton enters the charge separation region, it will leave after some time $\tau_{\text {acc }}$. The piston head is smoothly receding so protons enter the charge separation region at a rate of $n_{\mathrm{p}} v_{\mathrm{b}}$. After $\tau_{\text {acc }}$ protons will leave the charge separation region and the flux equilibrium can be established. There will, however, be a surplus of protons in the charge separation region equal to $n_{\mathrm{p}} v_{\mathrm{b}} \tau_{\mathrm{acc}}$. If this is much smaller than the number of protons in the charge separation region that are required for steady state force balance $\left(\left(n_{+} d_{+}\right)_{\text {st }}\right)$ then this is not significant, if not then the electric field cannot remain at its steady state value. The ratio of these two quantities can be estimated straightforwardly.

The magnitude of the spike in the electric field for steady state pistoning can be estimated from the condition for force balance on the electrons, $n_{+} d_{+}=n_{-} d_{-}$(charge conservation), and equation (20). Force balance on the electrons gives

$$
E_{x, 0}=\frac{2 I}{e n \_d_{-} c} \frac{1-\beta_{\mathrm{b}}}{1+\beta_{\mathrm{b}}} .
$$


Combining this with the other two expressions yields

$$
E_{x, 0}=\sqrt{\frac{2 I}{\epsilon_{0} c} \frac{1-\beta_{\mathrm{b}}}{1+\beta_{\mathrm{b}}}} .
$$

The acceleration time will therefore be given by

$$
\tau_{\mathrm{acc}}=\frac{2 m_{\mathrm{i}} v_{\mathrm{b}}}{e \sqrt{\left(2 I / \epsilon_{0} c\right)\left(\left(1-\beta_{\mathrm{b}}\right) /\left(1+\beta_{\mathrm{b}}\right)\right)}} .
$$

Using equations (20)-(23) it is found that the ratio $R=n_{\mathrm{p}} v_{\mathrm{b}} \tau_{\mathrm{acc}}>\left(n_{+} d_{+}\right)_{\mathrm{st}}$ is given by

$$
R=\frac{2 m_{\mathrm{p}} n_{\mathrm{p}} v_{\mathrm{b}}^{2}}{\epsilon_{0} \sqrt{\left(2 I / \epsilon_{0} c\right)\left(\left(1-\beta_{\mathrm{b}}\right) /\left(1+\beta_{\mathrm{b}}\right)\right)}}
$$

For the parameters considered in this paper, $R>0.8$ for all intensities. One should therefore not expect steady pistoning or an ideal phase space distribution for the protons. The maximum rate at which the electric field can grow during the 'ramp-up' phase of the saw-tooth oscillation is given by

$$
\frac{\mathrm{d} E_{x, 0}}{\mathrm{~d} t}=\frac{e n_{\mathrm{p}} v_{\mathrm{b}}}{\sqrt{2} \epsilon_{0}} .
$$

The factor of $\sqrt{2}$ accounts for the dwell time of a proton in the pure proton region (i.e. contribution to $n_{+} d_{+}$) not just the time it spends in the entire charge separation region. For the PIC simulation where $I=2 \times 10^{22} \mathrm{~W} \mathrm{~cm}^{-2}$, this predicts $\dot{E}_{x, 0}=3.8 \times 10^{28} \mathrm{~V} \mathrm{~m}^{-1} \mathrm{~s}^{-1}$. The grow rate of the saw-teeth in the simulation is about $\dot{E}_{x, 0}=2.4 \times 10^{28} \mathrm{~V} \mathrm{~m}^{-1} \mathrm{~s}^{-1}$ (figure 8).

The crash in the saw-teeth occurs when a proton bunch leaves the pure proton region. Proton bunching occurs due to the spatial and temporal variation of the spike in the electric field, which causes a series of 'wave-breaking' events similar to those described by Macchi et al [3]. Proton bunches leave the pure proton region on the ion acceleration time-scale, which is given by equation (23). For the PIC simulation where $I=2 \times 10^{22} \mathrm{~W} \mathrm{~cm}^{-2}$ this predicts a time between saw-tooth crashes of 5.2 fs. The PIC simulation results indicate that the crashes occur at intervals of about $6 \mathrm{fs}$.

\section{Conclusions}

In this paper a new analytic model for the 1D HP RPA problem has been developed. In the regime of large $\Xi\left(I / \rho c^{3}\right)$, which can be accessed with laser intensities in excess of $10^{21} \mathrm{~W} \mathrm{~cm}^{-2}$ and low- $\rho$ targets, the predictions of the purely non-relativistic analytic model and the new, fully relativistic model diverge significantly. In terms of the mean ion energy, and $\mathrm{HB}$ velocity, there is excellent agreement between the fully relativistic analytic model and 1D PIC simulations with constant laser intensity and circularly polarization in the high- $\Xi$ regime.

On the other hand, it was also found that although at lower intensities, illumination at constant intensity produced a quasi-monoenergetic beam, at high laser intensities the accelerated protons had a large energy spread. It is thought that this is due to a periodic 'overloading' of space-charge at the surface being driven by the laser pulse which in turn is due to the finite ion acceleration time.

\section{Acknowledgment}

The authors are grateful for the use of computing resources provided by Science and Technology Facilities Council's e-Science facility. 


\section{References}

[1] Wilks S, Kruer W, Tabak M and Langdon A B 1992 Phys. Rev. Lett. 691383

[2] Gibbon P 2005 Phys. Rev. E 72026411

[3] Macchi A et al 2005 Phys. Rev. Lett. 94165003

[4] Liseikina TV and Macchi A 2007 Appl. Phys. Lett. 91171502

[5] Macchi A 2006 Appl. Phys. B 82337

[6] Silva L et al 2004 Phys. Rev. Lett. 92 015002-1

[7] Chen M et al 2007 Phys. Plasmas 14113106

[8] Esirkepov T et al 2004 Phys. Rev. Lett. 92 175003-1

[9] Pegoraro F and Bulanov S V 2007 Phys. Rev. Lett. 99065002

[10] Robinson A P L et al 2008 New J. Phys. 10013021

[11] Zhang X et al 2007 Phys. Plasmas 14073101

[12] Akli KU et al 2008 Phys. Rev. Lett 100165002

[13] Denavit J 1992 Phys. Rev. Lett. 693052

[14] Estabrook K G, Valeo E J and Kruer W L 1975 Phys. Fluids 181151

[15] Kar S et al 2008 Phys. Rev. Lett. 100225004

[16] Yan X Q et al 2008 Phys. Rev. Lett. 100135003

[17] Klimo O et al 2008 Phys. Rev. ST-AB 11031301

[18] Marx G 1966 Nature 21122

[19] Simmons J F L and McInnes C R 1993 Am. J. Phys. 61205

[20] Zhang X et al 2007 Phys. Plasmas 14123108 